\title{
Mean platelet volume, neutrophil to lymphocyte ratio and platelet to lymphocyte ratio in determining the diagnosis or outcome in children with snakebite
}

\author{
Assist. Prof. Fesih Aktar ${ }^{a}$, Assist. Prof. Recep Tekin ${ }^{b}$
}

\begin{abstract}
Background: The objective of this study is to evaluate the relationships between the mean platelet volume (MPV), neutrophil to lymphocyte ratio (NLR) and platelet to lymphocyte ratio (PLR) variables and diagnosis or prediction of outcome in children with snakebite envenomation.

Methods: Children diagnosed with snakebite envenomation and a control group of healthy subjects were retrospectively evaluated. Patients were classified into three groups as mild, moderate and severe.

Results: 142 children were enrolled in the study. Leukocytosis $(\mathrm{p}=0.003)$, neutrophilia $(\mathrm{p}=0.026)$ and thrombocytopenia $(p=0.034)$ were significantly more common in severe snakebite envenomation, although no statistical significant were found in association with MPV, NLR and PLR between snakebite envenomation groups. The mean MPV, NLR and PLR were found to be significantly higher in children with snakebite compared to than among healthy controls.

Conclusions: Our results suggested that MPV, NLR and PLR may be useful for the diagnosis as inflammatory markers in snakebite envenomation.

Key words: Mean platelet volume; neutrophil-to-lymphocyte ratio; platelet-to-lymphocyte ratio; snake envenomation; child.

http:/ / dx.doi.org/10.5546/ aap.2017.eng.576
\end{abstract}

To cite: Aktar F, Tekin R. Mean platelet volume, neutrophil to lymphocyte ratio and platelet to lymphocyte ratio in determining the diagnosis or outcome in children with snakebite. Arch Argent Pediatr 2017;115(6):576-580. a. Dicle University School of Medicine, Department of Pediatric Infectious Disease, Diyarbakir, Turkey.

b. Dicle University School of Medicine, Department of Clinical Microbiology and Infectious Disease, Diyarbakir, Turkey.

E-mail address: Assist. Prof. Fesih Aktar: fesihaktar@yahoo.com

Funding: None.

Conflict of interest: None.

Received: 10-13-2016

Accepted: 6-7-2017

\section{INTRODUCTION}

There are no reliable diagnostic markers of snakebite available in clinical practice. ${ }^{1-3}$ Therefore, precise diagnosis of snakebite envenomation requires accurate identification of the snakebite and investigation of the clinical findings of envenomation. ${ }^{3}$ The laboratory signs of envenomation are derived from the severe cytotoxic, hematopoietic system and inflammatory effects of the venom. ${ }^{4,5}$ Snake venom aggravates systemic effects including inflammation and leukocyte proliferation at the bite site. Recent reports have suggested that novel laboratory parameters such as increased mean platelet volume (MPV), neutrophil to lymphocyte ratio (NLR), platelet to lymphocyte ratio (PLR), and thrombocyte count are associated with inflammation. ${ }^{4-6}$

No previous study has evaluated MPV, NLR and PLR in patients with snakebite. We hypothesized that snakebite envenomation, which is an inflammatory condition, may result in increases in systemic markers of inflammation such as MPV, NLR and PLR. The aim of this study was to evaluate the relationship between MPV, NLR, PLR and the diagnosis or prediction of outcome in children with snakebite envenomation.

\section{MATERIALS AND METHODS \\ Patients}

This retrospective study was performed in Pediatric Clinics and children that less than 18 years old included in the study. The medical records of all patients with a history of snakebite between June 2006 and September 2014 were obtained from the hospital records. The diagnosis of snakebite was made if the subjects had seen the snake or if the appearance of the puncture sites was clearly indicative of snakebite.

Patient data was obtained from patient followup cards completed at admission and the hospital records. Age, gender, severity of envenomation and laboratory data were collected. 


\section{Data collection and comparisons}

NLR and PLR were calculated as the ratio of neutrophils to lymphocytes and platelets to lymphocytes, respectively. Healthy subjects were children who applied to hospital for routine check-up. Children with any sign of infection, systemic illness or inflammation were excluded from the control group.

Patients were children with snakebite and an age and gender-matched control group of healthy children were enrolled in the study. $\mathrm{WBC}, \mathrm{Hb}$, neutrophile count, lymphocyte count, PLT, MPV, NLR and PLR values were compared between the two groups.

\section{Definitions}

The severity of envenomation was determined according to the criteria adapted by Otero. ${ }^{7}$ Mild envenomation is defined as swelling involving one or two segments of bitten limb and less than $4 \mathrm{~cm}$ increase in limb circumference with no bleeding in the bite site, no necrosis or systemic symptoms. Moderate envenomation is defined as swelling involving two or three segments of the bitten limb and more than $4 \mathrm{~cm}$ increase in limb circumference with local bleeding in the bite site, no local necrosis and minimal or no systemic symptoms. Severe envenomation results in swelling extending beyond the bitten limb (to trunk), blisters, local bleeding, necrosis or compartmental syndrome and clinical systemic signs including multiple systemic bleeding, hypotension or shock, disseminated intravascular coagulation, renal failure, cerebral hemorrhage or multi-systemic failure.

\section{Exclusion criteria}

Patients, with chronic disorders, anemia or other hematological diseases and acute bacterial infections were excluded. In addition, patients who did not see the snake and did not have bite marks of a snake or had any other forms of unknown bites not consistent with snakebites were excluded from the study.

The study protocol was approved by the Dicle University Medical School Ethics Committee.

\section{Statistical analysis}

All data analyses were performed using SPSS (Statistical Package for Social Sciences) version 18.0 program for Windows. The Kolmogorov-

TABLE 1. Demographic features and laboratory findings of snakebite severity groups

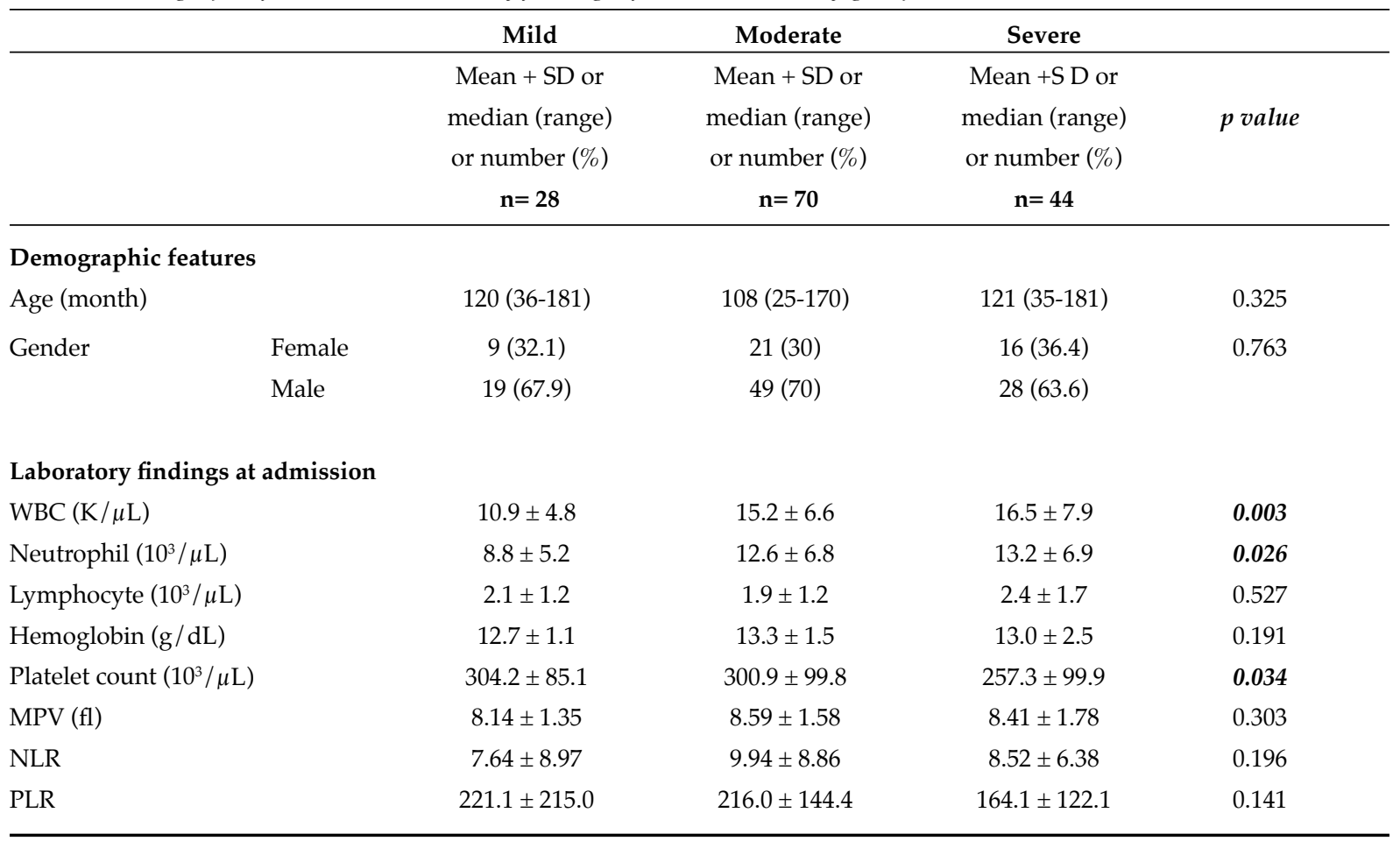

WBC: white blood cell, MPV: mean platelet volume, NLR: neutrophil to lymphocyte ratio, PLR: platelet to lymphocyte ratio, SD: standard deviation. 
Smirnov test was used to examine the normality distribution of continuous data. Normally distributed numerical variables were shown as mean plus/minus standard deviation. Normally distributed numeric variables were compared using the Student's T-test. The Chi-square test was used to compare categorical variables between the groups. One way ANOVA or Kruskal Wallis test was used for comparison between three subgroups. Cumulative incidence was calculated for the entire duration of the study, with 95\% confidence intervals. P-values of less than 0.05 were considered statistically significant.

\section{RESULTS}

142 children diagnosed with snakebite envenomation and 142 healthy subjects were retrospectively evaluated. The mean age of the patients was $110.1 \pm 37.5$ months and 96/142 $(67.6 \%)$ patients were male. The mean age of the control group was $97.8 \pm 44.3$ months and $95 / 142$ $(66.9 \%)$ were male. There were no significant differences in the mean age or gender distribution between the study and the control group ( $p>$ 0.05).

Twenty-eight $(19.8 \%)$ patients had mild snakebite envenomation, while $70(49.2 \%)$ had moderate snakebite envenomation and $44(31 \%)$ had severe snakebite envenomation. Leukocytosis $(p=0.003)$, neutrophilia $(p=0.026)$ and thrombocytopenia $(\mathrm{p}=0.034)$ were significantly more common in severe snakebite envenomation group, although no statistical significant were found in association with MPV, NLR and PLR $(\mathrm{p}>0.05)$ (Table 1).

The patient group exhibited significantly higher MPV, NLR, PLR, WBC, neutrophil and lymphocyte values and lower platelet count at admission compared to the control group $(\mathrm{p}<0.05)$ (Table 2).

\section{DISCUSSION}

In another study we found that duration of hospitalization, rural occurrence, WBC count, AST / ALT ratio, CK, hypoproteinemia, hypoalbuminemia, and hypocalcemia can be associated with the severity of snakebite. WBC and AST / ALT ratio can be used as follow-up criteria in children with snakebite. ${ }^{1}$ The present study indicated that MPV, NLR and PLR may be useful for the diagnosis as inflammatory markers of snakebite. To our knowledge, this is the first study investigating the relationship between snakebite and serum complete blood count parameters in children.

The venom has a complex structure including many toxins and proteins with systemic inflammatory, necrotizing and hemorrhagic effects and may account for several of the inflammatory manifestations observed in snakebite. ${ }^{4-6,8} \mathrm{MPV}$, NLR and PLR are measured as part of routine blood counts in many laboratories without additional cost. ${ }^{4,9}$. MPV is associated with thrombocyte activation and function under inflammatory conditions. ${ }^{6,9}$ Platelets are vital components of normal hemostasis

TABLE 2. Laboratory findings of study and control groups

\begin{tabular}{lccc}
\hline Hematological & Study group & Control group & \\
\hline Variables & $\begin{array}{c}\text { (n: 142) } \\
\text { Mean + SD or } \\
\text { median (range) }\end{array}$ & $\begin{array}{c}\text { (n: 142) } \\
\text { Mean + SD or } \\
\text { median (range) }\end{array}$ & value \\
\hline WBC $(\mathrm{K} / \mu \mathrm{L})$ & $14.9 \pm 7.05$ & & $<0.001$ \\
Platelet count $\left(10^{3} / \mu \mathrm{L}\right)$ & $287 \pm 99.3$ & $330 \pm 87.6$ & $<0.001$ \\
Neutrophil $\left(10^{3} / \mu \mathrm{L}\right)$ & $11.3(5.96-16.4)$ & $4.14(3.28-5.54)$ & $<0.001$ \\
Lymphocyte $\left(10^{3} / \mu \mathrm{L}\right)$ & $1.85(1.15-2.74)$ & $2.74(2.32-3.51)$ & $<0.001$ \\
MPV (fl) & $8.11(7.48-9.05)$ & $7.63(7.19-8.39)$ & 0.002 \\
NLR & $5.98(2.52-13.0)$ & $1.43(1.09-1.90)$ & $<0.001$ \\
PLR & $146.5(105.1-259.4)$ & $113.3(55.0-136.6)$ & $<0.001$ \\
\hline
\end{tabular}

WBC: white blood cell, MPV: mean platelet volume, NLR: neutrophil to lymphocyte ratio, PLR: platelet to lymphocyte ratio, SD: standard deviation. 
and they can release several inflammatory cytokines. ${ }^{4,6,9}$ MPV may increase in low-grade inflammation and may decrease in more severe inflammation due to the intensive consumption of platelets at inflammatory sites. ${ }^{4} \mathrm{MPV}$ is a reflection of proinflammatory conditions, where numerous inflammatory cytokines regulate inflammatory process. ${ }^{4}$ Snakebites involve substantial inflammatory activity, both locally and systemically. In the present study, significantly higher MPV was found among snakebite patients relative to healthy subjects. Therefore, we hypothesized that MPV may be useful for the diagnosis of snakebite.

MPV acts as an acute phase reactant in some inflammatory conditions depending on the severity of systemic inflammation. ${ }^{4,9}$. In the present study, we also compared the MPV values with the severity of disease, and found no relationship between MPV and disease severity among children with snakebites.

NLR is a marker of systemic inflammation. ${ }^{9,10}$ NLR is increased in inflammatory disease but has not been specifically evaluated in patients with snakebites. NLR is useful in diagnosis of inflammation. ${ }^{4,6,9-11 .}$. We found a significant relationship between NLR and snakebite envenomation relative to healthy control subjects. NLR may thus be an indicator of the inflammatory marker of snakebite.

NLR is also useful in the prediction of mortality and prognosis. NLR is a good predictor of disease severity in some illness. ${ }^{9-11}$ The mechanism underlying the association between NLR and disease severity has not been elucidated and remains controversial. Elevated neutrophil count may depend on activated IL- $1 \beta$, fever, neutrophilia, and the migration of neutrophils in to tissue. ${ }^{12}$ In present study, we found no relationship between NLR and the severity of disease in patients with snakebite.

Snakebite envenomation is associated with thrombosis, hemostasis and inflammation. ${ }^{13}$ PLR is another systemic inflammatory response indicator obtained from routine blood analysis, and can be used to evaluate the inflammatory process in snakebite. The number of platelets may increase under inflammatory conditions and bleeding, which can result in increased expression of inflammatory cytokines and thrombocytosis. ${ }^{6,11-13}$. Elevated platelet counts may reflect underlying inflammation and lower lymphocyte counts may represent uncontrolled inflammatory signaling. Hence, elevated PLR can be a useful marker of systemic inflammation. ${ }^{6,10}$. Some studies have reported that higher platelet and lower lymphocyte counts are associated with malignancies, coronary artery disease and various inflammatory diseases. ${ }^{9,14,15}$ Feng et al. reported that PLR was associated with poor survival in esophageal carcinoma. ${ }^{14}$ Acmaz et al. demonstrated elevated PLR in patients with endometrial cancer compared to control subjects and reported that this inflammatory marker could also be used in diagnosis..$^{15}$ In the present study, PLR was significantly higher in snakebite patients when compared to healthy control subjects. PLR has been reported associated with severity of disease, survival, differential diagnosis, prognosis and follow-up in several studies of various disease states..$^{9,13-15}$ We found no relationship between PLR and the severity of disease in the present study.

We have some potential limitations of this study, Our study was retrospective with short number of cases and performed in singleinstitution and the lymphocyte subtypes and other indicators of the inflammatory system such as cytokines, C-reactive protein and sedimentation were not investigated and the cardiac history of the patients was unremarkable.

\section{CONCLUSIONS}

MPV, NLR and PLR levels may be useful for the diagnosis of snakebite, which is sometimes difficult for pediatricians. We found no relationship between MPV, NLR, and PLR and snakebite severity in the present study. Hence, we concluded that MPV, NLR and PLR may not be reliable prognostic indicators in children with snakebite. But, futures investigations with a greater number of patients will be necessary to know if these biomarkers can be related to the severity of the clinical form in snakebite children.

\section{REFERENCES}

1. Aktar F, Aktar S, Yolbas I, et al. Evaluation of risk factors and follow-up criteria for severity of snakebite in children. Iran J Pediatr 2016;26(4):e5212.

2. Chippaux JP. Epidemiology of snakebites in Europe: a systematic review of the literature. Toxicon 2012;59(1): 86-99.

3. Gold BS, Dart RC, Barish RA. Bites of venomous snakes. N Engl J Med 2002;347(5):347-56.

4. Santhosh MS, Sundaram MS, Sunitha K, et al. Viper venominduced oxidative stress and activation of inflammatory cytokines: a therapeutic approach for over looked issues of snakebite management. Inflamm Res 2013;62(7):721-31.

5. Sunitha K, Hemshekhar M, Thushara RM, et al. Inflammation and oxidative stress in viper bite: an insight within and beyond. Toxicon 2015;98:89-97. 
6. Mannaioni PF, Di Bello MG, Masini E. Platelets and inflammation: role of platelet-derived growth factor, adhesion molecules and histamine. Inflamm Res 1997;46(1): $\pi 4-18$.

7. Otero-Patiño R. Epidemiological, clinical and therapeutic aspects of Bothrops asper bites. Toxicon 2009;54(7):998-1011.

8. Tekin R, Sula B, Cakır G, et al. Comparison of snakebite cases in children and adults. Eur Rev Med Pharmacol Sci 2015;19(14):2711-6.

9. Kemal Y, Yucel I, Ekiz K, et al. Elevated serum neutrophile to lymphocyte and platelet to lymphocyte ratios could be useful in lung cancer diagnosis. Asian Pac J Cancer Prev 2014;15(6):2651-4.

10. Bhat T, Teli S, Rijal J, et al. Neutrophil to lymphocyte ratio and cardiovascular diseases: a review. Expert Rev Cardiovasc Ther 2013;11(1):55-9.

11. Ulu S, Ulu MS, Bucak A, et al. Neutrophil-to-lymphocyte ratio as a new, quick, and reliable indicator for predicting diagnosis and prognosis of idiopathic sudden sensorineural hearing loss. Otol Neurotol 2013;34(8):1400-4.

12. Dinarello CA. Biologic basis for interleukin-1 in disease. Blood 1996;87(6):2095-147.

13. McCleary RJ, Kini RM. Snake bites and hemostasis/ thrombosis. Thromb Res 2013;132(6):642-6.

14. Feng JF, Huang Y, Chen QX. Preoperative platelet lymphocyte ratio (PLR) is superior to neutrophil lymphocyte ratio (NLR) as a predictive factor in patients with esophageal squamous cell carcinoma. World J Surg Oncol 2014;12:58.

15. Acmaz G, Aksoy H, Unal D, et al. Are neutrophil/ lymphocyte and platelet/lymphocyte ratios associated with endometrial precancerous and cancerous lesions in patients with abnormal uterine bleeding? Asian PacJCancer Prev 2014;15(4):1689-92.

\title{
Female authorship in Latin American pediatric journals
}

\author{
Paula Otero, M.D..$^{a, b}$, Cecilia Marcos ${ }^{b}$ and Fernando Ferrero, M.D. ${ }^{b, c}$
}

\begin{abstract}
Introduction. The participation of women in science increases every day. Here we estimated their participation in authorship in three Latin American pediatric journals indexed in PubMed. Methods. All articles published in 2015 in the Archivos Argentinos de Pediatría, the Jornal de Pediatría and the Revista Chilena de Pediatría were identified, and the first and last authors and the total number of authors by sex were determined.

Results. A total of 329 articles were identified. Out of 1432 authors, $59.9 \%$ were women. Also, $54.4 \%$ of all first authors and $48 \%$ of last authors were women. No significant difference was observed in female authorship ratio among the threejournals. Archivos Argentinos de Pediatría had a significantly lower number of women as first and last authors.

Conclusion. Women authorship ratio across three Latin American pediatric journals reached $59.9 \%$. Their role as first or last authors was significantly lower in the Archivos Argentinos de Pediatría.

Key words: Authorship, research report, women, periodic publications.
\end{abstract}

\footnotetext{
a. Hospital Italiano de Buenos Aires.

b. Archivos Argentinos de Pediatría.

c. Hospital General de Niños Pedro de Elizalde.

E-mail adress:

Fernando Ferrero M.D.:fferrero@intramed.net.
}

Funding: None.

Conflict of interest: None.

Received: 1-19-2017

Accepted: 6-12-2017 http: / / dx.doi.org/10.5546/ aap.2017.eng.580

To cite: Otero P, Marcos C, Ferrero F. Female authorship in Latin American pediatric journals. Arch Argent Pediatr 2017;115(6):580-583.

\section{INTRODUCTION}

The participation of women in the medical field has increased considerably in the $20^{\text {th }}$ century. As mentioned by Filardo, ${ }^{1}$ the female physicians ratio reaches 33\% in the United States of America, 47\% in the United Kingdom, and over $50 \%$ in Eastern Europe. The female physicians ratio is 50\% in Argentina (60\% among those younger than 40 years); ${ }^{2} 40 \%$ in Brazil ( $53 \%$ among those younger than 30 years), ${ }^{3}$ and $33 \%$ in Chile (46\% among those younger than 30 years). ${ }^{4}$ In the field of pediatrics, the participation of women is even more clear based on the number of female members of the Argentine Society of Pediatrics (9044/12 772, 70.8\%), the Brazilian Society of Pediatrics (16 372/ 22 158, 74\%), and the Chilean Society of Pediatrics (1393/2880, $48.3 \%$ ) (data obtained from the corresponding organizations in April 2017).

In spite of such apparent quantitative balance, there might still be differences in access to certain settings. For example, in Argentina, $73.1 \%$ of medicine students of Universidad de Buenos 